\title{
RAPID BOLD FMRI SIGNAL LOSS IN THE PRIMARY MOTOR CORTEX OF A STROKE PATIENT
}

\author{
Sonia M. Fioravanti Carvalho', Octavio M. Pontes-Neto', Soraia R.C. Fabio', \\ Joao P. Leite, Antonio Carlos Santos ${ }^{3}$, Draulio B. de Araujo ${ }^{1,2}$
}

Functional neuroimaging techniques have greatly contributed to better understand neurophysiological processes that occur after a brain insult. Among all techniques, functional magnetic resonance imaging (fMRI) has interesting advantages such as non-invasiveness and good spatial resolution. Therefore, fMRI has motivated a number of researchers to perform studies on brain recovery after stroke, most of which are based on blood oxygenation level dependent (BOLD) mechanism that rely on hemoglobin oxidative state changes. To perform BOLD-fMRI one needs to assume that the signal is stable throughout data acquisition'. However, many confounders may disturb such assumption, as disrupted neurovascular coupling in patients with cerebrovascular diseases ${ }^{2}$. To our knowledge, the stability of the BOLD signal in time during the execution of a task among blocks of a motor paradigm has never been studied during a single fMRI session, although it is of important concern in clinical application of $\mathrm{FMRI}^{3}$.

We report rapid changes in $\mathrm{FMRI} B O L D$ signal in a stroke patient, which may have correlation to the altered neurophysiological processes associated with this condition.

\section{CASE}

We studied a 71 years old male patient who suffered an ischemic stroke in 1993, in the right middle cerebral artery (MCA) territory. During the acute phase, the patient showed marked left hemiparesis (not able to maintain effort against gravity). He recovered partially, developing moderate hemiparesis (able to maintain some effort against gravity). Ischemia on the MCA territory was evaluated by CT and MRI. Neurological deficits were appraised through the NIHSS scale and functional evaluation accomplished by Barthel Index, ARA test and Fugl Meyer Scale (FMS).

MR images were acquired in a 1.5T scanner (Siemens, Magneton Vision), in three sessions, in different days. The functional data set was acquired with EPI-BOLD sequences (TR/TE $=4500 / 66.0$ $\mathrm{ms}, \mathrm{FA}=90^{\circ}$, voxel volume $\left.=1.64 \mathrm{~mm} \times 1.64 \mathrm{~mm} \times 5.00 \mathrm{~mm}\right)$. Also a high-resolution T1-weighted image $\left(1 \mathrm{~mm}^{3}\right)$ was acquired using a $3 \mathrm{D}$ fast spoiled gradient echo ( $T R / T E=9.7 / 4.0 \mathrm{~ms}, \mathrm{FA}=12^{\circ}$ ).

A block paradigm was designed with six blocks of 27 seconds of rest, and five blocks of activity (27 seconds), when the patient was cued to open and close his hand. The patient performed a "dry run" to ensure task compliance, and to guarantee a stable frequency of movement throughout the exam, as it may be an exhausting task for a person with a residual hand motor deficit. fMRI analysis was performed in Brain Voyager QX (version 1.6; Maastricht, the Netherlands), using the general linear model. Prior to statistical analysis, data was corrected for movement artifact, scanner drift and slice scan time.

This study was approved by the local ethic committee and the patient gave written informed consent after having carefully been informed about the study.

\section{DISCUSSION}

As revealed by MRI and CT, the infarct affected the territory of a major branch of the right MCA, including the angular, central and pre-central gyrus. NIHSS score, in the period of the first fMRI was 3, what reveals that the patient had significant neurological and functional recovery, with reasonable grade of independence, reaching 85 points at Barthel Index (BI), considered functionally independent. Moreover, the patient showed low scores in FMS and ARA test, mostly related to hand movements, revealing a residual motor deficit.

Figure shows the $\mathrm{fMRI}$ activation related to each hand tested independently. The unaffected hand showed activation over the contralateral primary motor cortex (MT), pre-motor cortex (PM) and supplementary motor area (SMA). The paretic hand showed a broader pattern of activation, involving ipsilateral M1, PM and SMA.

Figures 1A, 1C, 1D and 1F show the time evolution of the $B O L D$ response throughout the exam. The green col-

\section{PERDA RÁPIDA DO SINAL BOLD NO CÓRTEX MOTOR PRIMÁRIO EM PACIENTE COM AVC}

'Department of Neurology, Psychiatry and Clinical Psychology, Faculdade de Medicina de Ribeirão Preto, Ribeirão Preto SP, Brazil (FMRP); ${ }^{2}$ Department of Physics and Math, FFCLRP; ${ }^{3}$ Department of Internal Medicine, FMRP. This study was supported by the Brazilian funding agencies FAPESP, CNPq and CAPES.

Received 18 February 2008, received in final form 6 August 2008. Accepted 6 September 2008

Dr. Draulio B. de Araujo - DFM / FFCLRP / University of São Paulo - 14040-901 Ribeirão Preto SP - Brasil. E-mail: draulio@usp.br 


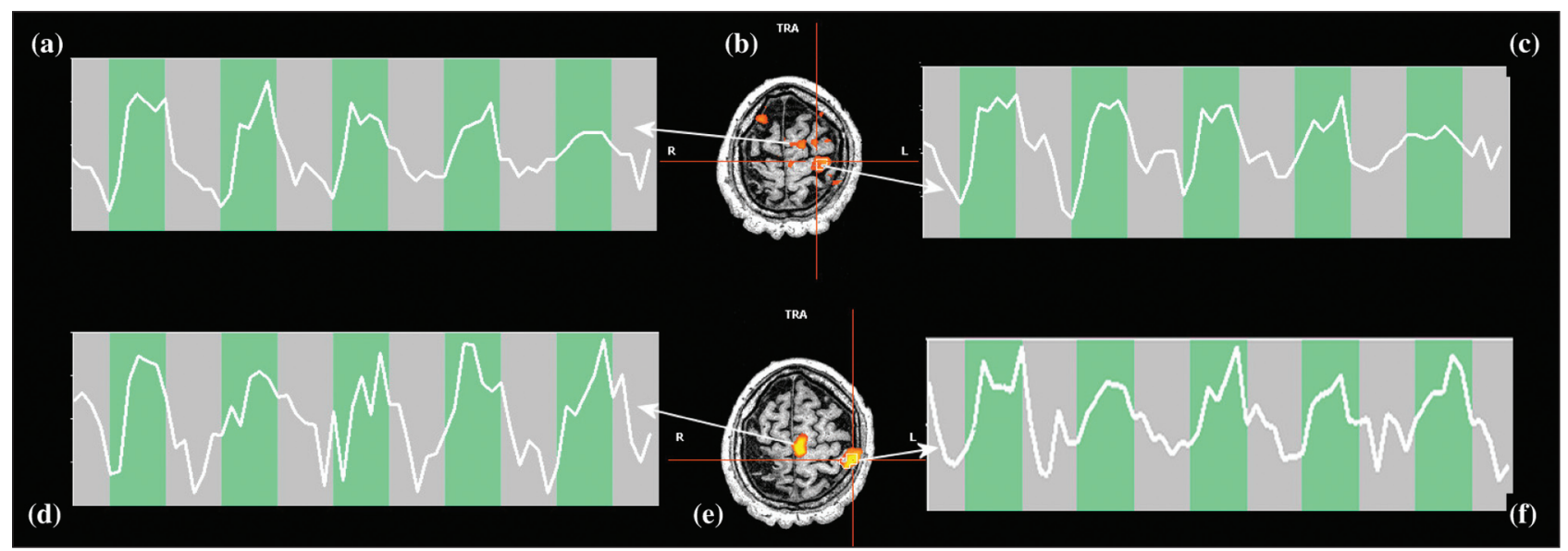

Figure. The first row $(A, B$, and $C)$ shows results from the paretic hand, and the second row $(D, E$, and $F)$ shows the results from the unaffected one. The unaffected (right) hand shows contralateral activation in M1, PM and SMA. The activation of the paretic hand is reorganized on different ipsilateral cortical motor areas (MI, PM, and SMA). The progressive decline of BOLD activation amplitude is showed in (A) and (B), compared with the unaffected hand in (D) and (F).

umns represent periods when the patient was performing the task, whereas gray columns represent periods of rest. The patient did not show any sign of synkinesia. There was a clear decrease in the BOLD amplitude along the course of the exam (Fig 1A and 1C). Conversely, the BOLD response from the unaffected hand showed a normal stable temporal evolution (Fig 1D and 1F).

In general, two activation patterns have been observed in post stroke patients: reorganization of the motor cortex to lesion boundaries, or its migration to homologue motor areas of the unaffected hemisphere ${ }^{4}$. Besides the standard activation observed herein, a remarkable rapid decrease of $\mathrm{FMRI}$ signal was presented in the reorganized motor areas (Fig 1A and 1C). The BOLD signal in the pre-frontal area, as well as SMA, reduces significantly throughout the fMRI exam. In opposition, its amplitude is stable when the patient is performing the task with the unaffected hand (Fig ID and 1F). Although some studies have reported decrease BOLD signal, most are related to amplitude variation in different $\mathrm{fMRI}$ sessions. Unlike the results presented in the literature, the present study illustrates a BOLD signal loss during a single fMRI session.

Some hypothesis may explain the observed phenomena. We herein speculate about three possible ones: muscular weakness, impaired neurovascular coupling, and hyperventilation. Nevertheless, any of these causes for the BOLD amplitude reduction would preclude from correctly detecting such activation pattern by using standard analysis methods, as general linear model.

When a movement is accomplished, the first output by the motor cortex refers to the contraction force. It is well known that damage of brain tissue following stroke affects corticospinal and other supraspinal motor pathways. The reduction in neural traffic results in motor neuron loss and disruption of these primary force control mechanisms. Consequently, it affects the fast recruitment of spinal motoneurons, resulting in slower movements and decreased peak of strength range. Previous studies of motor unit activity in hemiplegic patients have suggested that motor unit firing rates tend overall to be decreased relative to ipsilesional limbs, and reduced firing rates may affect the capacity to produce fused contraction ${ }^{5}$. Indeed, previous $\mathrm{fMRI}$ studies have demonstrated that a similar effect would be correlated with changes in M1 BOLD amplitude, but not in SMA, where activity would be stable 6 . Moreover, an fMRI study of a simple force modulation task has shown that the amplitude change of the M1 BOLD signal is directly correlated to the force applied while SMA is stable ${ }^{7}$. In summary, activation of SMA seems to be independent on the duration and motor strength of the stimulus $s^{6,7}$. In our study we observe BOLD amplitude reduction on both M1 and SMA.

Another hypothesis to be considered is a possible altered neurovascular coupling, already mapped in cerebrovascular diseases ${ }^{8}$. The BOLD signal is based in local changes of cerebral blood volume, cerebral blood flow (CBF) and blood oxygenation. Therefore, disturbance in physiological conditions can influence the coupling between neuronal activation and the hemodynamic response. After an ischemic stroke, the reactivity of the cerebral circulation to vasomotor stimuli is altered, autoregulation is impaired, and the increase in CBF produced by functional activation is decreased ${ }^{8}$. In stroke such coupling may be disrupted, and may be responsible for the observed BOLD decrease.

Last of all, recently fMRI techniques have been used to detect $\mathrm{rCBF}$ by means of BOLD signal changes, during hy- 
percapnia or hyperventilation'. During hyperventilation, the decrease in $\mathrm{PCO}_{2}$ leads to vasoconstriction and consequently a decrease in rCBF. Hence, the condition increases the local concentration of deoxyhemoglobin, leading to a slightly decrease in the local MR signal. Therefore, if a patient presents some hyperventilation, as a result of tiredness, the BOLD signal would decrease throughout task execution ${ }^{10}$.

Overall, this study discloses the existence of an altered BOLD response in a stroke patient. We have suggested that such change may be caused by fatigue, impaired neurovascular coupling or hyperventilation. This important observation raises the question of whether shortterm BOLD modification can prevent activation from being detected in pathological conditions, such as stroke.

\section{REFERENCES}

1. Araujo DB, Tedeschi W, Santos AC, Elias J, Neves UPC, Baffa O. Shannon entropy applied to the analysis of event-related fMRI time series. Neuroimage 2003;20:311-317.
2. Rossini PM, Altamura C, Ferretti A, et al. Does cerebrovascular disease affect the coupling between neuronal activity and local haemodynamics? Brain 2004;127:99-110.

3. Escorsi-Rosset S, Wichert-Ana L, Bianchin MM, et al. Variable fMRI activation during two different language tasks in a patient with cognitive delay. Arq Neuropsiquiatr 2007;65:985-987.

4. Cao Y, D'Olhaberriague L, Vinkingstad EM, Levine SR, Welch KM. Pilot study of functional MRI to assess cerebral activation of motor function after post stroke hemiparesis. Stroke 1998;20:112-122.

5. Gemperline JJ, Allen S, Walk D, Rymer WZ. Characteristics of motor unit discharge in subjects with hemiparesis. Muscle Nerve 1995;18: 1101-1114.

6. Birn RM, Saad ZS, Bandettini PA. Spatial heterogeneity of the nonlinear dynamics in the fMRI BOLD response. Neuroimage 2001;14:817-826.

7. Peck KK, Sunderland A, Peters AM, Butterworth S, Clark P, Gowland PA. Cerebral activation during a simple force production task: changes in the time course of the haemodynamic response. NeuroReport 2001;12:2813-2816.

8. Iadecola C. Neurovascular regulation in the normal brain and in Alzheimer's disease. Nature Rev Neurosci 2004;5:347-360.

9. Andrade KC, Pontes-Neto OM, Santos AC, Baffa O, Leite JP, Araujo DB. Quantitative aspects of brain perfusion dynamic induced by breathholding BOLD fMRI. Arq Neuropsiquiatr 2006;64:895-898.

10. Leoni RF, Mazzeto-Betti KC, Andrade KC, Araujo DB. Quantitative evaluation of hemodynamic response after hypercapnia among different brain territories by fMRI. Neuroimage 2008;41:1192-1198. 